\title{
Variability and Performance of Short to Long-Range Single Baseline RTK GNSS Positioning in Indonesia
}

\author{
Irwan Gumilar ${ }^{1, *}$, Brian Bramanto ${ }^{1}$, Fuad F. Rahman ${ }^{1}$ and I Made D. A. Hermawan ${ }^{2}$ \\ ${ }^{1}$ Geodesy Research Group, Institut Teknologi Bandung, Indonesia \\ ${ }^{2}$ General Technology Indonesia
}

\begin{abstract}
As the modernized Global Navigation Satellite System (GNSS) method, Real Time Kinematic (RTK) ensures high accuracy of position (within several centimeters). This method uses Ultra High Frequency (UHF) radio to transmit the correction data, however, due to gain and power issues, Networked Transport of RTCM via Internet Protocol (RTCM) is used to transmit the correction data for a longer baseline. This Research aims to investigate the performance of short to long-range single baseline RTK GNSS (Up to $80 \mathrm{KM}$ ) by applying modified LAMBDA method to resolve the ambiguity in carrier phase. The RTK solution then compared with the differential GNSS network solution. The results indicate that the differences are within RTK accuracy up to $80 \mathrm{~km}$ are several centimeter for horizontal solution and three times higher for vertical solution.
\end{abstract}

\section{Introduction}

Global Navigation Satellite System or commonly abbreviated as GNSS is a satellite based point or moving object positioning system. In simplified, GNSS determines the user position by measuring at least four distance from known satellites position simultaneously. Point position can be determined by either using absolute or relative point positioning. Absolute point positioning uses stand-alone receiver GNSS while relative point positioning use two receivers GNSS on the same time observation as base and rover, which can also be referred as differential positioning. There are various other methods that can be used in GNSS point positioning, such as static and kinematic method. Static is such condition when the object is not moving, while kinematic is when the object is moving. The position of those condition can be determine by using either absolute or differential positioning. The point position can also be determine either real time or post-processing.

Real Time Kinematic (RTK) has been widely used is several application [1-4]. Moreover, with the existence of CORS (Continuously Operating Reference System), the RTK can be done easier. CORS is a reference system that works continuously as a base station in differential GNSS positioning. CORS send various kind of correction data needed in GNSS measurement using a specific data transfer system, such as RTCM (Radio Technical Commission for Maritime Services). CORS can provide a position with an accuracy of $\pm 20 \mathrm{~mm}$, however, it should be noted that the optimum distance between CORS station and rover is about $15-20 \mathrm{~km}$ [56]. This is due to various types of errors that will increase with increasing baseline distance.
In RTK GNSS measurements, the distance between base station and rover might varies from several kilometers to hundreds of kilometers. Along with the greater baseline, the greater error also occurred on the observation data, such as ionospheric bias, tropospheric bias and orbital error bias, which lead to unresolved ambiguity integer.

In this study a new algorithm [7] is used to improve the RTK performance in long baseline condition. The new algorithm determine the atmospheric bias and correct the orbital error by using precise orbit that obtained from Starfire ${ }^{\mathrm{tm}}$ network and further process in resolving ambiguity integer.

\section{Research Methodology}

\subsection{Tropospheric Bias Determination}

In general, atmospheric bias (Ionospheric and Tropospheric) is estimated through the Kalmam Filter process along with the position, velocity and ambiguity parameters. Tropospheric bias thus can be overcome using a variety models such as UNB, Hopfield or Saastomoinen Models [8-10]. Dry components of the troposphere can be easily modeled, however, the wet components are relatively hard to modeled.

Various tropospheric condition can be overcome by using a scale factor for the Zenith delay value. After removing the tropospheric delay with the model from the observed data, the Relative Tropospheric Zenith Delay (RTZD) can be approximate properly.

Corresponding author: igumilar@gd.itb.ac.id 


\section{2 lonospheric Bias Determination}

Having implemented the broadcast model and Double Difference (DD) process to minimize the ionospheric delay, the residual ionospheric delay are then estimated on the Kalman filter calculation. Empirically, the residual ionospheric delay can be modeled as $1^{\text {st }}$ order Gauss Markov. Ionospheric correction derived from global and regional ionospheric model (for example WAAS) can be considered as virtual measurements and included in the Kalman filter estimation. However, the accuracy obtained from both of broadcast and WASS models are still not enough. This is because the model can only handle some part of the ionosphere delay.

In recent year, the concepts of using a network reference station for GNSS RTK have been developed by several academic institutions and several GNSS manufacturers. The development of these concepts resulted new correction for atmospheric bias and orbital errors for GNSS measurements. This correction can be used to overcome errors which are influenced by the length of baseline. The developed ionosphere model will improve the performance and reliability of determining ambiguity and reduce the time to get fix solutions on RTK GNSS.

\subsection{Orbital Error}

Navigation message broadcast by satellites contain orbit keplerian elements and time elements. These message is estimated from network GNSS observation station and control segment of GNSS that is updated every two hours. Several test have proven that the error results obtained from orbital errors might vary from 2 to 5 meters [11]. In general, orbital error is the biggest source of error in RTK GNSS point positioning after removing the atmospheric error.

Some of the differential GNSS systems have been able to provide the orbital correction, such as HA-ND GPS (High Accuracy Nationwide Differential GPS). HA-ND GPS is a differential correction broadcasting system that developed by several US government organization. John Deer also has developed the Starfire ${ }^{\text {tm }}$ system. This system sends orbital corrections in a global scale via communication satellites.

\subsection{Ambiguity Fixing}

In theory, ambiguity is a constant value, however, float ambiguity uncertainty values that is estimated through the Kalman filter may contain multipath and systematic bias errors that are not modeled, such as orbital errors and atmospheric bias residual. Based on these considerations, before the ambiguity value is fixed, the ambiguity for the L1/L2 signal and its variance were first converted into L1/Wide Lane (WL) form which is then returned to L1/L2 after fulfilling the ambiguity criteria test. This process can be called as a Partial Search and Fix process. This process determined the "true" ambiguity value. This process is carried out when the conventional ambiguity search process does not meet the criteria.
In ambiguity fixing process, a minimum of five satellites is needed. When there are six or more satellites observed, some ambiguity values from the satellite can be removed in the search process. By calculating all the five satellite combinations needed, the value of ambiguity that has the biggest bias can be seen and eliminated so as to facilitate the process of determining ambiguity. This process increases the success rate of determining ambiguity, but this process takes a lot of time and it will be very difficult to determine all satellite combinations with limited tool processing capabilities.

Therefore, the Partial Search and Fix process is used. After the DD calculation process for both frequencies is carried out, the ambiguity values and its variances can be obtained through the Kalman filter process. Although the parameters used in the process of ambiguity resolution are ambiguities of L1 and L2, the search process using the Lambda method can be different. So that the forms L1 and L2 can be changed into L1 and (WL). WL is used because it has a longer wavelength $(0.86 \mathrm{~cm})$ so it will be easier to determine its ambiguity. From the search process using the Lambda method, the best and second best ambiguity candidate values are obtained. If the value meets the ambiguity validation test, the best value is used in the calculation of fixed solutions. But if it does not meet the validation test, the Partial Search and Fix process can be done by eliminating the satellite with the value of ambiguity outside the best and second best value.

\subsection{Data acquisition}

Nine point were observed using GNSS RTK in this research. The number of points is adjusted to the needs of the baseline distance interval to be observed and then adjusted to the availability of reference points in the field. The baseline length varies from $5 \mathrm{~km}$ to $85 \mathrm{~km}$ with an interval for approximately every $10 \mathrm{~km}$. The observation point and its baseline length can be seen on Table 1. and Fig. 1.

Table 1. Point ID and its baseline length

\begin{tabular}{|c|c|}
\hline Point ID & Baseline length \\
\hline BDG066 & $5.00 \mathrm{~km}$ \\
\hline BDG073 & $10.00 \mathrm{~km}$ \\
\hline ITB01 & $17.70 \mathrm{~km}$ \\
\hline TTG698 & $21.45 \mathrm{~km}$ \\
\hline KM30 & $30.00 \mathrm{~km}$ \\
\hline TTG693 & $36.34 \mathrm{~km}$ \\
\hline TTB & $47.85 \mathrm{~km}$ \\
\hline J681A & $59.78 \mathrm{~km}$ \\
\hline N1.0304 & $72.57 \mathrm{~km}$ \\
\hline J670 & $83.51 \mathrm{~km}$ \\
\hline
\end{tabular}




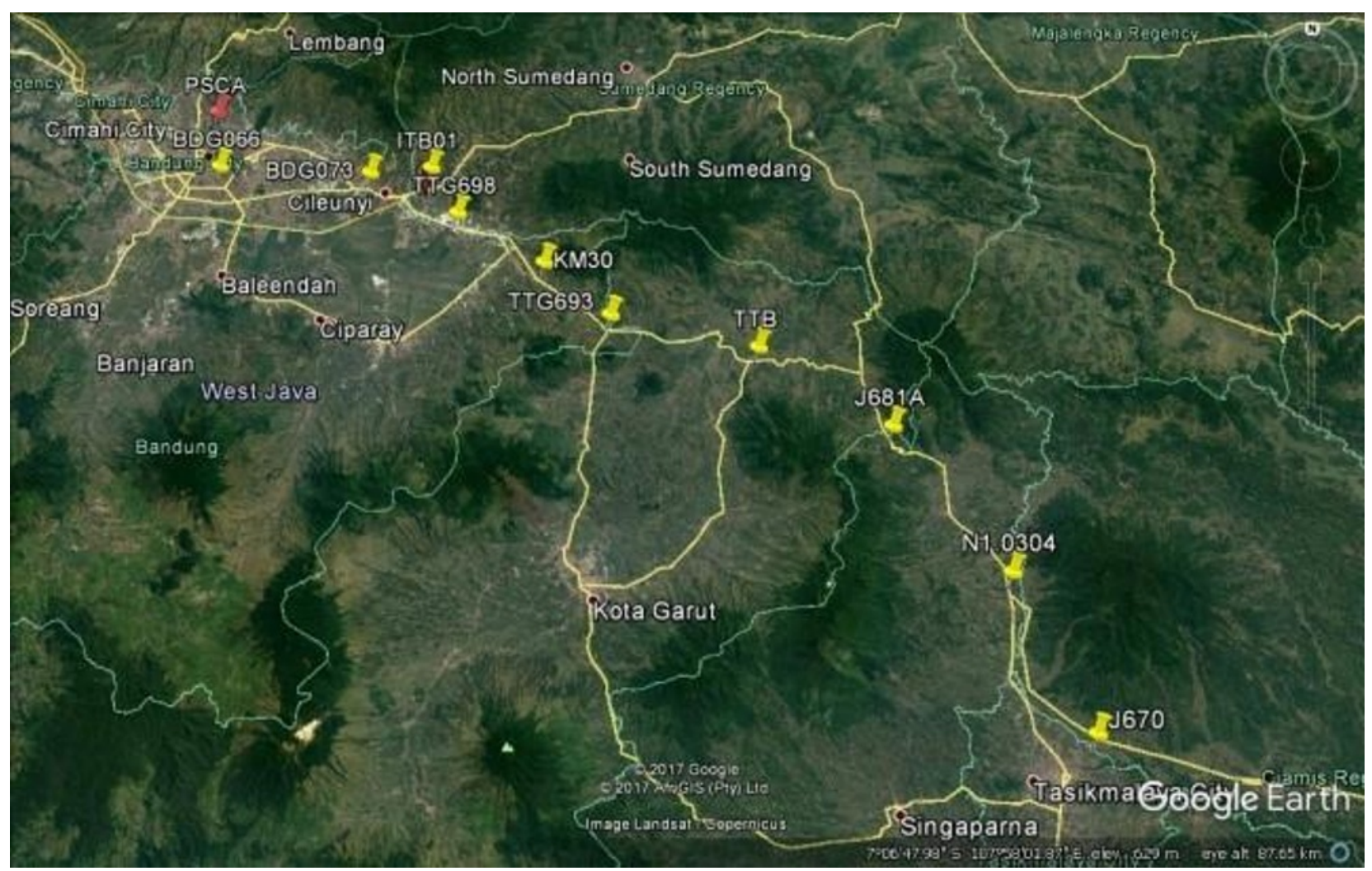

Fig. 1. Location of the observed point

\section{Results and Discussions}

\subsection{Accuracy and Precision}

To assess the accuracy of point positioning, the estimated point position from RTK GNSS were compared to reference coordinate. The reference coordinate were derived from static differential method. The precision is derived from RTK GNSS position deviation from its mean position. Three selected observation points will be discussed further in this section. Three selected observation points are ITB01, TTB and J670.

The maximum error of ITB01 for easting and northing components are about $24 \mathrm{~cm}$ and 10 respectively, while maximum error for height component is $28 \mathrm{~cm}$. The precision for easting, northing and the elevation components are about $3 \mathrm{~cm}, 2.6 \mathrm{~cm}$ and $12 \mathrm{~cm}$.
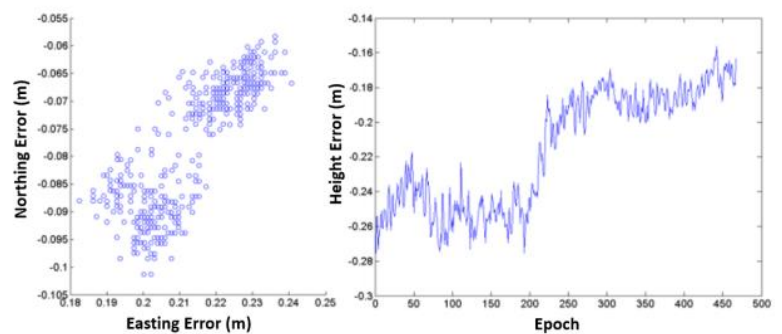

Fig. 2. Position error of ITB01
The high value of error likely due to the geometry of the satellite. To illustrate the different condition, a comparison observation point is needed. BDG066 is chosen because BDG066 has relatively good obstruction compared with ITB01. Fig. 3. shows the skyplot of BDG066 and ITB01. There is no observed data in the direction of $30^{\circ}$ to $120^{\circ}$ from the North of ITB01, while BDG066 has a good skyview for all of the direction.

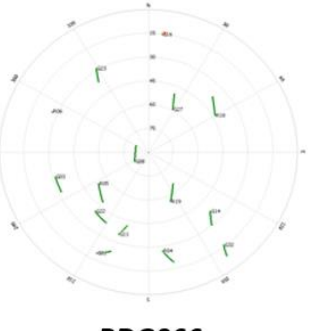

BDG066

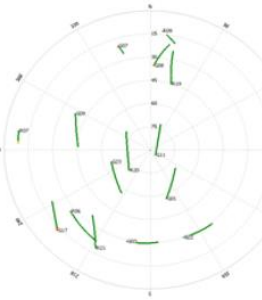

ITB01
Fig. 3. Skyplot of BDG066 and ITB01 


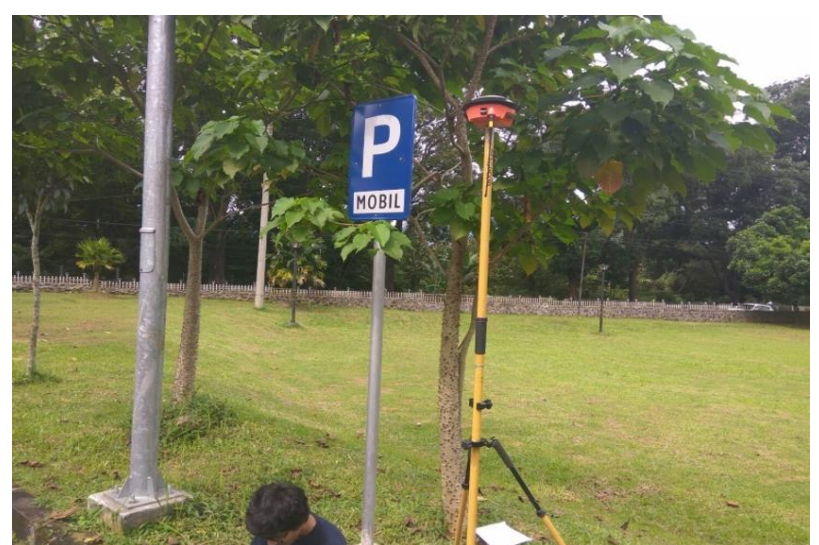

Fig. 4. ITB01 observation point

The maximum error of TTB for easting and northing components are no more than $6.5 \mathrm{~cm}$ and 5.5 respectively, while for height component is vary from 5 $\mathrm{cm}$ to $26 \mathrm{~cm}$. The precision for easting, northing and the elevation components are about $3 \mathrm{~cm}, 5.8 \mathrm{~cm}$ and $13 \mathrm{~cm}$.

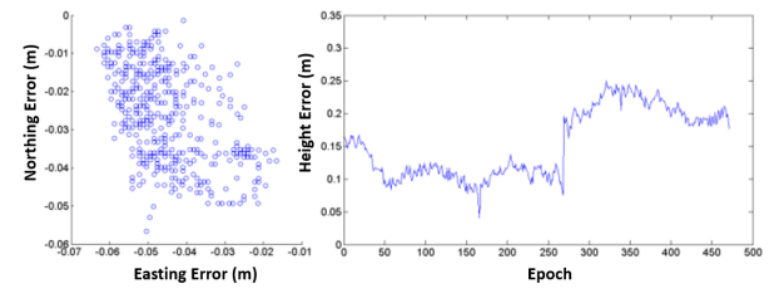

Fig. 3. Position error of TTB

With the baseline length for about $83.51 \mathrm{~km}, \mathrm{~J} 670$ was the farthest observation point. The maximum error of ITB01 for easting and northing components are about $8 \mathrm{~cm}$ and 5 respectively, while maximum error for height component is $18 \mathrm{~cm}$. The precision for easting, northing and the elevation components are about $3.7 \mathrm{~cm}, 3.7 \mathrm{~cm}$ and $15 \mathrm{~cm}$.

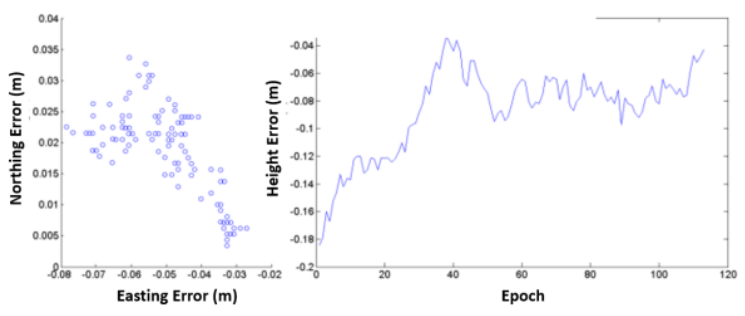

Fig. 3. Position error of J670

\subsection{Overal Accuracy}

Fig.4. and Fig. 5. show the overall accuracy both of horizontal and vertical estimated position. The accuracy for horizontal varies from $2 \mathrm{~cm}$ to $45 \mathrm{~cm}$ while the accuracy of vertical varies from $10 \mathrm{~cm}$ to $70 \mathrm{~cm}$. The huge error mostly due to the unresolved bias.

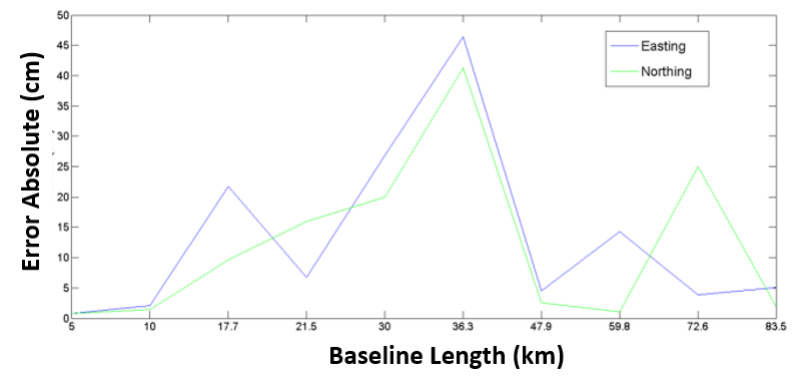

Fig. 4. Overall horizontal accuracy

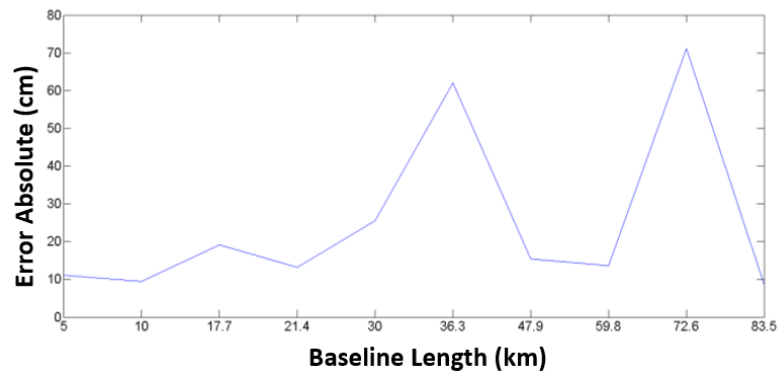

Fig. 4. Overall vertical accuracy

In GNSS data acquisition, there are numerous factors that can affect the estimated position. One of them is field condition. In accordance with previous explanation in Section 2, that error on the GNSS RTK observation will increase along with the increase of the baseline length. With a greater baseline length, there will be spatial and temporal variation in the atmospheric bias. Therefore, in order to be able to analyze the effects of spatial and temporal variations, the weather conditions and the observation time are needed.

Table 2. Point ID and its weather condition

\begin{tabular}{|c|c|c|c|}
\hline \multirow{2}{*}{ Point ID } & \multicolumn{2}{|l|}{ Time observation } & \multirow{2}{*}{$\begin{array}{l}\text { Weather } \\
\text { Condition }\end{array}$} \\
\cline { 2 - 4 } & Start & End & \\
\hline BDG066 & $10: 30$ & $11: 15$ & Drizzle \\
\hline BDG073 & $20: 05$ & $21: 15$ & Daylight \\
\hline ITB01 & $12: 10$ & $13: 15$ & Daylight \\
\hline TTG698 & $14: 50$ & $15: 25$ & Daylight \\
\hline KM30 & $18: 42$ & $19: 00$ & Daylight \\
\hline TTG693 & $16: 35$ & $16: 55$ & Drizzle \\
\hline TTB & $18: 40$ & $19: 05$ & Daylight \\
\hline J861A & $09: 35$ & $10: 15$ & Drizzle \\
\hline N1.0304 & $11: 00$ & $12: 00$ & Daylight \\
\hline J670 & $13: 55$ & $15: 30$ & Daylight \\
\hline
\end{tabular}

The huge error on ITB01 (17.7 km), TTG693 $(36.34 \mathrm{~km})$ and J861A $(59.78 \mathrm{~km})$ are due to the unresolved atmospheric bias and ionosphere bias. As 
seen on Table 2, the observation time of ITB01 was on the noon, the high activity on the ionosphere and with the error addition from the poor geometry satellites, lead into a worse estimation coordinate. TTG693 and J861 A suffer a variation on the atmospheric condition, combined with the baseline length, the estimated position is going worse.

\subsection{Reliability Analysis}

Reliability means how consistent the method produces fixed solutions. In this case, the reliability value is obtained from the comparison of the RTK Fixed (Dual) solution with all solutions obtained during the observation time of each baseline. The percentage results of each baseline can be seen in Fig. 5.

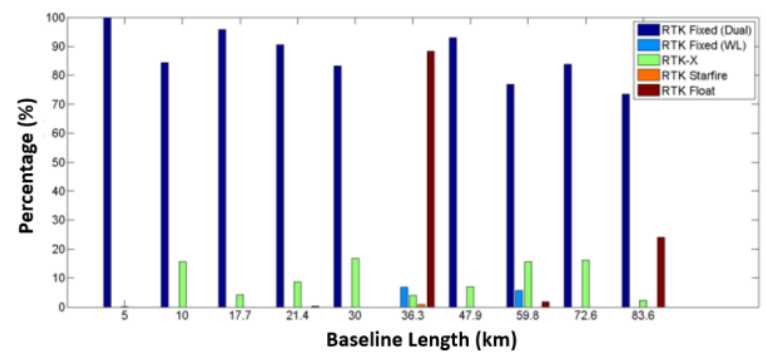

Fig. 5. Percentage for each solution

TTG693 has the lowest percentage of fixed dual solution compared to the others. TTG693 suffer a bad obstruction condition. It has a lot of vegetation. In this condition, ambiguity fixing is very difficult to obtain so that it takes time to the first ambiguity fixing. Overall, the ambiguity fixing percentage is decreasing along with the increasing of the baseline length as seen on Fig. 6.

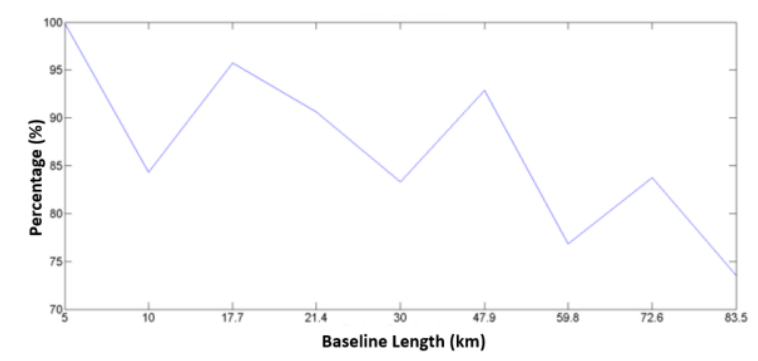

Fig. 6. Ambiguity fixing percentage over baseline length

\section{Conclussions}

The GNSS RTK measurement results show the error position and precision for each baseline length. The error accuracy for easting components vary from $0.8 \mathrm{~cm}$ to $46.6 \mathrm{~cm}$, while for northing components vary from 0.8 $\mathrm{cm}$ to $41.3 \mathrm{~cm}$. Error accuracy for height components vary from $8.6 \mathrm{~cm}$ to $62.1 \mathrm{~cm}$. The results also indicate that rate of ambiguity fixing depends on the baseline length and the site obstruction..

\section{References}

1. Tae-Suk Bae and Minho Kim, "Performance Analysis of Network-RTK Techniques for Drone Navigation considering Ionospheric Conditions," Journal of Sensors, vol. 2018, Article ID 5154697, 8 pages, 2018. https://doi.org/10.1155/2018/5154697

2. W. Stempfhuber and M. Buchholz, "A Precise, Low-Cost RTK GNSS System For UAV Applications" International Archives of the Photogrammetry, Remote Sensing and Spatial Information Sciences, Volume XXXVIII-1/C22, 2011

3. S. R. Saghravani, Sa'ari bin Mustapha and S. F. Saghravani "Accuracy Comparison of RTK-GPS and Automatic Level for Height Determination in Land Surveying" Journal Of Reviews and Surveys, Volume 1 Issue 1, 2009

4. Ahmed EL-MOWAFY "Surveying with GPS for Construction Works Using the National RTK Reference Network and Precise Geoid Models" $1^{\text {st }}$ FIG International Symposium on Engineering Surveys for Construction Works and Structural Engineering, 2004

5. Yanmin Feng and Jinling Wang "GPS RTK Performance Characteristics and Analysis" Journal of Global Positioning Systems 2008 Vol. 7, No. 1 : $1-8,2008$

6. R. M. Alkan, İ. M. Ozulu and V.İlÇi "Comparison of Single Baseline RTK and Network RTK GNSS Methods" International Symposium On Modern Technologies, Education And Professional Practice In Geodesy And Related Fields, 2017

7. Liwen Dai, Dan Eslinger and Tenny Sharpe "Innovative Algorithms tp Improve Long Range RTK Reliability and Availability" ION NTM 2007

8. S. Katsougiannopoulos, C. Pikridas, D. Rossikopoulos, I. M. Ifadis and A. Fotiou "Tropospheric Refraction Estimation Using Various Models, Radiosonde Measurements and Permanent GPS Data" FIG Congress XXIII, 2006

9. Chalermchon Satirapod and Prapod Chalermwattanachai "Impact of Different Tropospheric Models on GPS Baseline Accuracy: Case Study in Thailand" Journal of Global Positioning Systems (2005) Vol. 4, No. 1-2: 36-40, 2005

10. Langley, R.B. "Propagation of the GPS Signals, In: Kleusberg, A. and Teunissen, P.J.G. (eds), GPS for Geodesy (2nd edition)", Springer-Verlag, Berlin Heidelberg New York, 111-150, 1998

11. Janusz Zielinski and Ryszard Zdunek "GPS orbit accuracy and its influence on large network solutions" Advances in Space Research 23(4):665665 DOI: 10.1016/S0273-1177(99)00149-0, 1999 